\title{
TITLE:
}

\section{Optimal wavelet expansion via sampled-data control theory}

$\operatorname{AUTHOR}(\mathrm{S})$ :

Kashima, Kenji; Yamamoto, Yutaka; Nagahara, Masaaki

\section{CITATION:}

Kashima, Kenji ... [et al]. Optimal wavelet expansion via sampled-data control theory. IEEE SIGNAL PROCESSING LETTERS 2004, 11(2): 79-82

\section{ISSUE DATE:}

2004-02

URL:

http://hdl.handle.net/2433/50278

\section{RIGHT:}

(c)2004 IEEE. Personal use of this material is permitted. However, permission to reprint/republish this material for advertising or promotional purposes or for creating new collective works for resale or redistribution to servers or lists, or to reuse any copyrighted component of this work in other works must be obtained from the IEEE. 


\section{Optimal Wavelet Expansion via Sampled-Data Control Theory}

Kenji Kashima, Student Member, IEEE, Yutaka Yamamoto, Fellow, IEEE, and Masaaki Nagahara, Member, IEEE

\begin{abstract}
Discrete wavelet transform is usually executed by the so-called pyramid algorithm. It, however, requires a proper initialization, i.e., expansion coefficients with respect to the basis of one of the desired approximation subspaces. An interesting question here is how we can obtain such coefficients when only sampled values of signals are available. This letter provides a design method for a digital filter that (sub-)optimally gives such coefficients assuming certain a priori knowledge on the frequency characteristic of target functions. We then extend the result to the case of nonorthogonal wavelets. Examples show the effectiveness of the proposed method.
\end{abstract}

Index Terms-Discrete wavelet transform (DWT), pyramid algorithm, sampled-data control.

\section{INTRODUCTION}

$\mathbf{C}$ OMPUTATION of the discrete wavelet transform (DWT) of a signal begins by approximation by projecting it onto one of the subspaces spanned by shifted scaling functions, constituting multiresolution analysis. This determines the finest resolution, and one is led to find expansion coefficients of coarser orders from there. The pyramid algorithm [10] can then be invoked to obtain such lower scale approximation coefficients. Furthermore, this can be implemented by a multirate filter bank [8], [10], [11], [16]. In this procedure, one has to first find expansion coefficients with respect to the basis formed by the shifted scaling functions. The subsequent approximation accuracy in the pyramid algorithm depends on these coefficients.

In digital signal processing, however, it is common practice that only sampled values of signals are available. Then it is not possible to accurately compute such expansion coefficients in the first place. One may then directly employ the sampled values as such coefficients. This is convenient, but obviously not satisfactory from the theoretical point of view, and hence referred to as a "wavelet crime" by Strang and Nguyen [10].

For this problem, Delyon and Juditsky [6] gave an error analysis in the Besov space, a subspace of a Sobolev space, but filters which perform better than the wavelet crime are not discussed. $\mathrm{Pu}$ and Francis [9] gave an optimal filter based on the $H^{\infty}$ criterion, assuming a certain frequency domain characteristic for target signals. On the other hand, their filter is neither causal nor finite-dimensional, and hence not easily realizable.

Manuscript received January 9, 2003; revised February 17, 2003. The associate editor coordinating the review of this manuscript and approving it for publication was Dr. Paulo Goncalves.

The authors are with the Department of Applied Analysis and Complex Dynamical Systems, Graduate School of Informatics, Kyoto University, Kyoto 606-8501, Japan.

Digital Object Identifier 10.1109/LSP.2003.821664

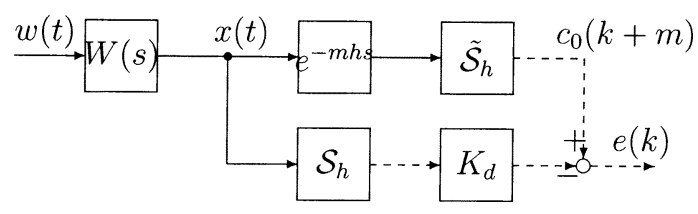

Fig. 1. Error system.

We propose instead to use a finite-dimensional and causal (with a finite-time delay) digital filter; for its design, we assume that the scaling function has compact support, and employ sampled-data control theory as per [7], [13]-[15], etc. While the performance may deteriorate compared to that in [9], it is easily realizable and, moreover, as we see later, it can be extended to nonorthogonal wavelets.

The letter is organized as follows. We first formulate our problem in the $H^{\infty}$ sampled-data control setting. We then reduce this to an equivalent discrete-time $H^{\infty}$ problem. These results are further extended to the case of oversampled signals and nonorthogonal scaling functions. The optimal filter is compared with the conventional wavelet crime to exhibit the difference.

\section{PRoblem Formulation}

We work with a multiresolution analysis (MRA) $\left\{\mathcal{V}_{j}\right\}_{j \in \mathbf{Z}}$ and a scaling function $\phi$ [8], [10]. Then $\mathcal{V}_{j}$ is spanned by $\left\{\phi_{j, k}\right\}_{k \in \mathbf{Z}}$ where $\phi_{j, k}(t):=2^{j / 2} \phi\left(2^{j} t-k h\right)$. Therefore for $x(t) \in L^{2}(\mathbf{R})$, its orthogonal projection onto $\mathcal{V}_{j}$

$$
v_{j}(t)=\sum_{k \in \mathbf{Z}} c_{j}(k) \phi_{j, k}
$$

can be viewed as an approximation of $x(t)$ at scale $j$. The scale of resolution increases as we move up in the nest.

Our problem here is to compute $\left\{c_{J}(k)\right\}_{k \in \mathbf{Z}}$ for a given integer $J \geq 0$, directly from these samples $\{x(k h)\}_{k \in \mathbf{Z}}$ via digital filters. This problem is called the optimal initialization problem of the DWT and, for the following case of orthogonal wavelets, is formulated and solved by giving an optimal solution in [9].

Assumption 1: The scaling function $\phi$ is orthonormal, i.e.,

$$
\langle\phi(t), \phi(t-k h)\rangle_{L^{2}(\mathbf{R})}=\delta_{k, 0}
$$

for all $k \in \mathbf{Z}$. Here $\delta_{i j}$ denotes the Kronecker delta.

This assumption makes $\left\{\phi_{J, k}\right\}_{k \in \mathbf{Z}}$ an orthonormal system of $\mathcal{V}_{J}$ and the projection coefficients are given by the inner product $\left\langle x, \phi_{J, k}\right\rangle_{L^{2}(\mathbf{R})}$.

We first formulate the design problem of a digital filter for optimal initialization for the case of $J=0$. Consider the block diagram Fig. 1. The weighting function $W(s)$ is a rational and 
proper lowpass filter which governs the frequency domain characteristic of target functions. For a given scaling function $\phi$, the operator $\tilde{\mathcal{S}}_{h}$ is defined by

$$
\tilde{\mathcal{S}}_{h}: L^{2}(\mathbf{R}) \longrightarrow \ell^{2}(\mathbf{Z}): x(t) \mapsto\left\{c_{0}(k)\right\}_{k \in \mathbf{Z}}
$$

The operator $\mathcal{S}_{h}$ denotes the sampler with sampling period $h$. The digital filter $K_{d}$ is to be designed. The upper path gives the expansion coefficients for the delayed signal whereas the lower path gives an approximant resulting from the sampler and digital filter $K_{d}(z)$. Denote by $\mathcal{T}_{e w}$ the operator from $w(t)$ to the error $\{e(k)\}$. The objective here is to minimize the worst case error, i.e.,

$$
\left\|\mathcal{T}_{\text {ew }}\right\|=\sup _{w \in L^{2}(\mathbf{R})} \frac{\left\|\mathcal{T}_{e w} w\right\|_{\ell^{2}(\mathbf{Z})}}{\|w\|_{L^{2}(\mathbf{R})}}
$$

We reformulate the problem as follows. First note that

$$
\left(\tilde{\mathcal{S}}_{h} x\right)(k)=\mathcal{S}_{h}(\mathbf{F} x)(k) .
$$

Here $\mathbf{F}$ denotes the continuous-time linear time-invariant system with impulse response $\breve{\phi}(t):=\phi(-t)$. This means that the problem above is an optimal discretization problem of noncausal continuous-time system $\mathbf{F}$. In view of this, we make yet another assumption.

Assumption 2: The allowable delay step $m$ and the scaling function $\phi(t)$ satisfy that $\phi(t)$ has compact support contained in $[0, m h]$, i.e.,

$$
\phi(t)=0, t \notin[0, m h] .
$$

Many well-known wavelets have compact support, for example, Haar scaling function and B-splines. With this assumption, the error system shown in Fig. 1 becomes causal. Our design problem then becomes as follows.

Problem 1: Given a stable $W(s)$, a prespecified bound $\gamma>$ 0 , an integer $m$, and scaling function $\phi$ satisfying Assumptions 1 and 2 , find a causal and finite dimensional digital filter $K_{d}(z)$ such that $\left\|\mathcal{T}_{\text {ew }}\right\|<\gamma$.

\section{Reduction to Finite-Dimensional Problem}

Suppose $m=1$ for simplicity; the same method applies to other cases. Introduce state space realizations

$$
\begin{aligned}
W(s) & =C(s I-A)^{-1} B, \\
K_{d}(z) & =C_{K}\left(z I-A_{K}\right)^{-1} B_{K}+D_{K} .
\end{aligned}
$$

A difficulty in Problem 1 is that the error system contains both continuous- and discrete-time dynamics. To avoid this, we apply the lifting operator [1], [12]

$$
\mathcal{L}: L^{2}(\mathbf{R}) \rightarrow \ell^{2}{ }^{2}[0, h)(\mathbf{Z}): w(t) \mapsto\{\tilde{w}[k](\theta)\}_{k \in \mathbf{Z}}
$$

to continuous-time signals in Fig. 1 , where $\tilde{w}[k](\theta):=w(k h+$ $\theta)$. We then have, from (1), that

$$
\left(\tilde{\mathcal{S}}_{h} x\right)(k)=\int_{0}^{h} \phi(\tau) \tilde{x}[k](\tau) d \tau .
$$

The lifting operator $\mathcal{L}$ preserves norms and the error system $\mathcal{T}_{\text {ew }} \mathcal{L}^{-1}:\{\tilde{w}[k](\theta)\}_{k \in \mathbf{Z}} \mapsto\{e(k)\}_{k \in \mathbf{Z}}$ can be rewritten as the following discrete-time dynamics:

$$
\begin{aligned}
{\left[\begin{array}{c}
X_{c}(k+1) \\
X_{d}(k+1)
\end{array}\right] } & =A_{T}\left[\begin{array}{l}
X_{c}(k) \\
X_{d}(k)
\end{array}\right]+\left[\begin{array}{c}
\mathcal{B} \\
0
\end{array}\right] \tilde{w}[k] \\
e(k) & =C_{T}\left[\begin{array}{l}
X_{c}(k) \\
X_{d}(k)
\end{array}\right]+\mathcal{D} \tilde{w}[k]
\end{aligned}
$$

where

$$
\begin{aligned}
A_{T} & :=\left[\begin{array}{cc}
e^{A h} & 0 \\
B_{K} C & A_{K}
\end{array}\right] \\
C_{T} & :=\left[\begin{array}{lll}
\int_{0}^{h} C e^{A \theta} \phi(\theta) d \theta-D_{K} C & -C_{K}
\end{array}\right] \\
\mathcal{B} & : \tilde{w}(\tau) \mapsto \int_{0}^{h} e^{A(h-\tau)} B \tilde{w}(\tau) d \tau \\
\mathcal{D} & : \tilde{w}(\tau) \mapsto \int_{0}^{h} \int_{0}^{\theta} C e^{A(\theta-\tau)} B \phi(\theta) \tilde{w}(\tau) d \tau d \theta .
\end{aligned}
$$

Here the input signal space $L^{2}[0, h)$ is infinite dimensional. On the other hand, $\mathcal{B}$ and $\mathcal{D}$ are finite-rank operators. Therefore, invoking their adjoint operators([3], [4]), the problem reduces to a finite-dimensional one.

Theorem 1: $\left\|\mathcal{T}_{\text {ew }}\right\|<\gamma$ if and only if

$$
\left\|C_{T}\left(z I-A_{T}\right)^{-1}\left[\begin{array}{c}
B_{T} \\
0
\end{array}\right]+D_{T}\right\|_{\infty}<\gamma
$$

where $B_{T}$ and $D_{T}$ are matrices defined as

$$
L L^{T}:=\left[\begin{array}{c}
\mathcal{B} \\
\mathcal{D}
\end{array}\right]\left[\begin{array}{ll}
\mathcal{B}^{*} & \mathcal{D}^{*}
\end{array}\right] \quad\left[\begin{array}{c}
B_{T} \\
D_{T}
\end{array}\right]:=L .
$$

This theorem means that Problem 1 is reducible to a oneblock $H^{\infty}$ - control problem. This is easily and effectively solvable via dhinflmi in the LMI toolbox of MATLAB [2]. For $m>1$, we can apply the same method by lifting the scaling function by (2). The degree of the obtained filter does not exceed $n+m$, where $n$ is the degree of $W$.

\section{EXTENSIONS}

We now give some generalizations, including the case of nonorthogonal wavelets.

\section{A. Projection Onto Higher Resolution Subspace}

We have given a procedure to optimally initialize the wavelet expansion, i.e., optimally projecting onto $\mathcal{V}_{0}$ which has the same scale of resolution as the sampling rate. It is, however, possible to project onto a subspace with a higher scale of resolution than $\mathcal{V}_{0}$, by making use of a given frequency distribution (i.e., weighting $W$ ) of the original signals. This leads us to the design problem depicted in Fig. 2.

The differences with Problem 2 are $\tilde{\mathcal{S}}_{h / 2^{J}}$ and $\uparrow 2^{J}$. First, define $\tilde{\mathcal{S}}_{h / 2^{J}}$

$$
\tilde{\mathcal{S}}_{h / 2^{J}}: L^{2}(\mathbf{R}) \longrightarrow \ell^{2}(\mathbf{Z}): x(t) \mapsto\left\{c_{J}(k)\right\}_{k \in \mathbf{Z}}
$$

and the output of upper path is $c_{J}(k)$. In accordance with this, the sampled values are also upsampled by $\uparrow 2^{J}$ to match with the sampling rate of the upper path and processed by $K_{d}(z)$. This gives an approximation problem in the space $\mathcal{V}_{J}$ instead of $\mathcal{V}_{0}$. 


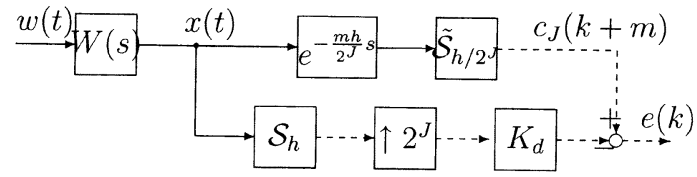

Fig. 2. Error system.

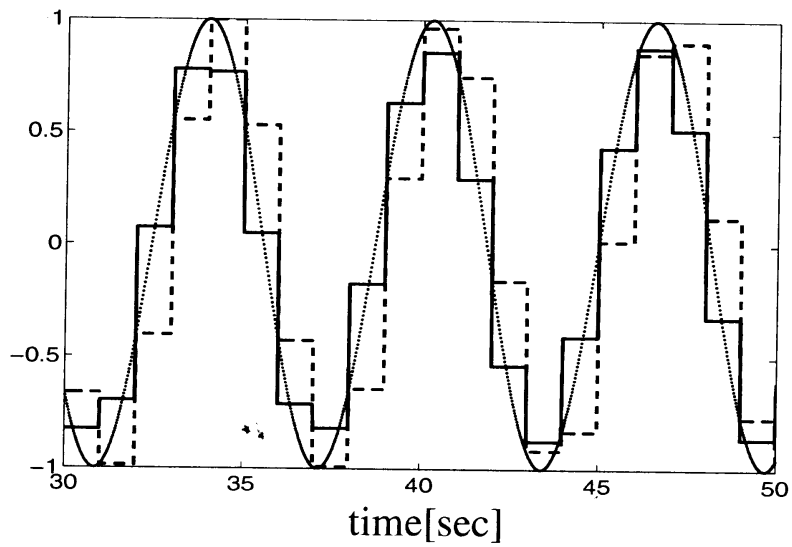

Fig. 3. Time response. (Dotted line) Source signal. (Dashed line) Wavelet crime. (Solid line) Proposed.

A difficulty here is that the overall error system is multirate one, but one can reduce this problem to a single-rate problem via discrete-time lifting [4], [5], [13], [14].

\section{B. Nonorthogonal Scaling Function}

When Assumption 1 is not satisfied, taking the inner product with the scaling function does not directly give projection coefficients. In such a case we can employ the dual basis $\left\{\xi_{k}(t)\right\}_{k \in \mathbf{Z}}$ such that

$$
\left\langle\phi_{0, p}, \xi_{q}\right\rangle_{L^{2}(\mathbf{R})}=\delta_{p, q}
$$

According to MRA, we can take

$$
\xi_{k}(t)=\sum_{k} g(k) \phi(t-k), k \in \mathbf{Z}
$$

for the dual basis. Here, desirable coefficients given by $\left\langle x, \xi_{k}\right\rangle_{L^{2}(\mathbf{R})}$ are represented as

$$
c_{0}(k)=\sum_{l} g(l) c_{0}^{\prime}(k+l), k \in \mathbf{Z}
$$

where $c_{0}^{\prime}(k)=\left\langle x, \phi_{J, q}\right\rangle_{L^{2}(\mathbf{R})}$. Truncating the sum above suitably to approximate the expansion, we can obtain $c_{0}(k)$ along with $c_{0}^{\prime}(k)$ via FIR digital filter

$$
K_{L}(z)=\sum_{l=l_{s}}^{l_{f}} g(l) z^{l-l_{f}} .
$$

For $c_{0}^{\prime}(k)$ we can apply the method in the previous section.

\section{NuMERICAL EXAMPLE}

We present an example for the case of the Haar and secondorder B-spline wavelets. Take the sampling period $h=1$ and weighting function $W(s)$, let

$$
W(s)=\frac{1}{(T s+1)(0.1 T s+1)}
$$

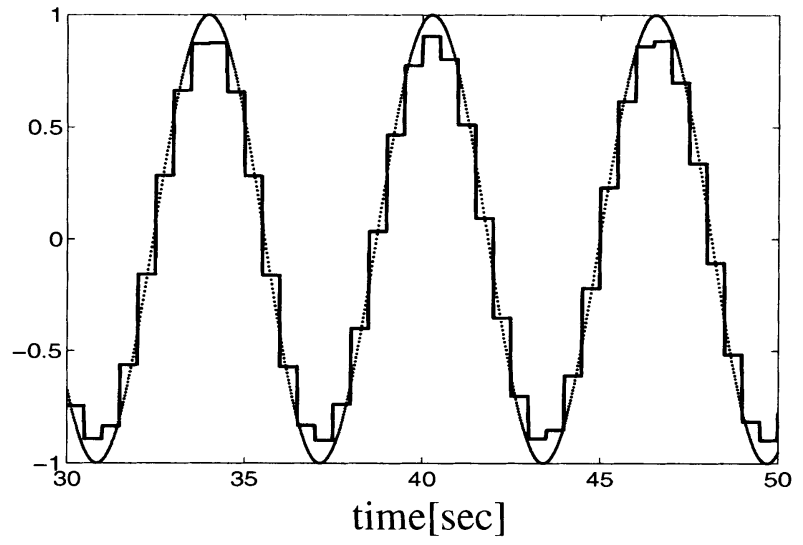

Fig. 4. Time response. (Dotted line) Source signal and (solid line) proposed (projection onto $\mathcal{V}_{1}$ )

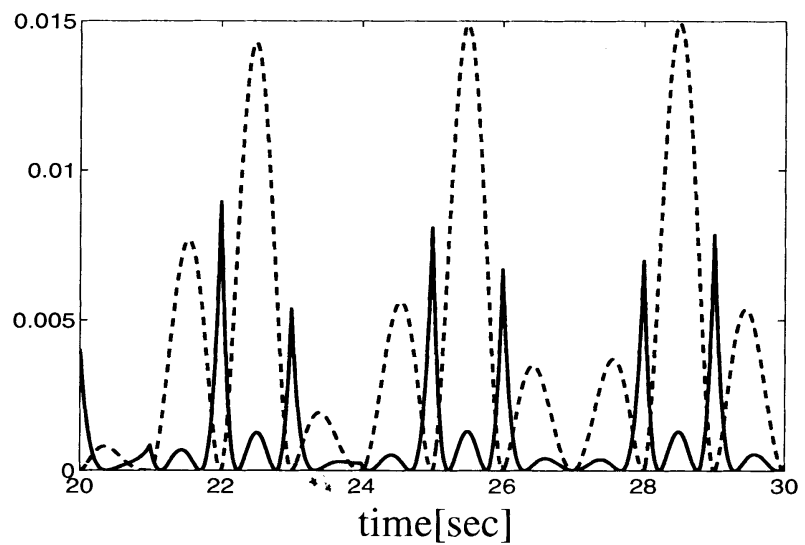

Fig. 5. Time responses of errors. (Dashed line) Wavelet crime and (solid line) projection onto $\mathcal{V}_{0}$.

where $T:=22.05 / \pi$. This simulates a fairly wide-range orchestral music frequency energy distribution observed in many commercial CDs with sampling rate $44.1 \mathrm{kHz}$.

We first consider the Haar wavelet and take delay step $m=1$. Note that this satisfies Assumptions 1 and 2. The error norm $\left\|\mathcal{T}_{e w}\right\|$ obtained by Theorem 1 is -35.4622 (decibels), which is nearly equal to the approximately computed infimum by [9], i.e., the case of an infite-dimensional and noncausal filter. On the other hand, that of the wavelet crime is -23.7590 (decibels).

Figs. 3 and 4 show the time responses of the proposed methods and the wavelet crime against the source signal $x(t)=\sin t$. Fig. 3 shows the projection onto $\mathcal{V}_{0}$ whereas Fig. 4 shows the projection onto $\mathcal{V}_{1}$, per the method given in Section IV-B.

Now consider the second-order B-splines. We take $m=2$ to satisfy Assumption 2. Note, however, that this does not satisfy Assumption 1 and its corresponding subspaces consist of piecewise linear functions. For this scaling function the dual basis is given by (5), where $g(l)=\sqrt{3}(\sqrt{3}-2)^{|l|}$. Here we take

$$
K_{L}(z)=\sum_{l=-3}^{3} g(k) z^{l-3}
$$

and the total delay is 5 steps. The corresponding squared errors for $x(t)=\sin t$ are shown in Fig. 5 .

Both examples exhibit quite an admirable performance. 


\section{CONCLUDING REMARKS}

We have presented a method to design a realizable (sub-)optimal filter for the optimal initialization problem of the DWT under some assumptions, based on sampled-data control theory. We have also extended the results to the higher order expansion and to the nonorthogonal wavelets. The numerical examples show that the obtained filters perform better than the wavelet crime.

\section{REFERENCES}

[1] B. Bamieh, J. B. Pearson, B. A. Francis, and A. Tannenbaum, "A lifting technique for linear periodic systems with applications to sampled-data control systems," Syst. Control Lett., vol. 17, pp. 79-88, 1991.

[2] P. Gahinet, A. Nemirovski, A. J. Laub, and M. Chilali, LMI Control Toolbox: The Math Works, 1995.

[3] T. Chen and B. A. Francis, "On the $\mathcal{L}^{2}$-induced norm of a sampled system," Syst. Control Lett., vol. 15, pp. 211-219, 1990.

[4] — Optimal Sampled-Data Control Systems. Berlin, Germany: Springer, 1995.

[5] — "Design of multirate filter banks by $H_{\infty}$ optimization," IEEE Trans. Signal Processing, vol. 43, pp. 2822-2830, Dec. 1995.

[6] B. Delyon and A. Juditsky, "On the computation of wavelet coefficients," J. Approx. Theory, vol. 88, no. 1, pp. 2822-2830, 1996.
[7] P. P. Khargonekar and Y. Yamamoto, "Delayed signal reconstruction using sampled-data control," in Proc. 35th Conf. Decision and Control, 1996, pp. 1259-1263.

[8] S. Mallat, A Wavelet Tour of Signal Processing. New York: Academic, 1998.

[9] Pu. Qian and B. A. Francis, "Optimal initialization of the discrete wavelet transform," in Proc. Workshop Recent Advances in Control, 1998.

[10] G. Strang and T. Nguyen, Wavelet and Filter Banks. Cambridge, U.K.: Wellesley-Cambridge, 1997.

[11] P. P. Vidyanathan, Multirate Systems and Filter Banks. Englewood Cliffs, NJ: Prentice-Hall, 1993

[12] Y. Yamamoto, "New approach to sampled-data systems: A function space method," in Proc. 29th Conf. Decision and Control, 1990, pp. $1881-1887$

[13] Y. Yamamoto, H. Fujioka, and P. P. Khargonekar, "Signal reconstruction via sampled-data control with multirate filter banks," in Proc. 36th Conf. Decision and Control, 1997, pp. 3395-3400.

[14] Y. Yamamoto and P. P. Khargonekar, "From sampled-data control to signal processing," in Learning, Control and Hybrid Systems, 1998, vol. 241, Springer Lecture Notes in Control and Information Sciences, pp. 108-126.

[15] Y. Yamamoto, M. Nagahara, and H. Fujioka, "Multirate signal reconstruction and filter design via sampled-data $H^{\infty}$ control," in Proc. MTNS2000, 2000.

[16] G. Zelniker and F. J. Taylor, Advanced Digital Signal Processing. New York: Marcel Dekker, 1994 